\title{
Binge Drinking Affects Brain Oscillations Linked to Motor Inhibition and Execution
}

López-Caneda, E. ; Rodríguez Holguín, S. ${ }^{2}$, Correas, A.3 ; Carbia, C. ${ }^{2}$; González-Villar, A. ${ }^{2}$; Maestú, F.3 ${ }^{3}$ Cadaveira, F. ${ }^{2}$

${ }^{1}$ Neuropsychophysiology Lab, Research Center on Psychology (CIPsi), School of Psychology, University of Minho, Braga, Portugal

${ }^{2}$ Department of Clinical Psychology and Psychobiology, University of Santiago de Compostela, Galicia, Spain

${ }^{3}$ Laboratory of Cognitive and Computational Neuroscience, Centre of Biomedical Technology (CTB), Madrid, Spain

Introduction: Neurofunctional studies have shown that binge drinking (BD) patterns of alcohol consumption during adolescence and youth are associated with anomalies in brain functioning. Recent evidence suggests that event-related oscillations (EROs) may be an appropriate index of neurofunctional damage associated with alcoholism. However, there is no study to date that has evaluated the effects of $\mathrm{BD}$ on oscillatory brain responses related to task performance. The purpose of the present study was to examine brain oscillations linked to motor inhibition and execution in young binge drinkers (BDs) compared to age-matched controls. Methods: Electroencephalographic activity was recorded from 64 electrodes while 72 university students (36 controls and 36 BDs) performed a visual Go/NoGo task. EROs along with the Go-P3 and NoGo-P3 eventrelated potential (ERP) components were analysed. Results: While no significant differences between groups were observed regarding ERPs, ERO analysis showed that BDs displayed a lower oscillatory response than controls in delta and theta frequency ranges during Go and NoGo conditions. Conclusions: Findings are congruent with ERO studies showing reduced delta and/or theta oscillations in alcoholics during Go/NoGo tasks. Thus, BDs appear to show disruptions in neural oscillations linked to motor inhibition and execution similar to those observed in alcohol-dependent subjects. Finally, these results are the first to evidence that oscillatory brain activity may be a sensitive indicator of underlying brain anomalies in young BDs, which could complement standard ERP measures. 
Key words Alcohol, Binge Drinking, Event-Related Potentials, Event-Related Oscillations, Response Inhibition.

Corresponding author: Eduardo López Caneda; e-mail: eduardo.lopez@usc.es; Neuropsychophysiology Lab, Research Center on Psychology (CIPsi), School of Psychology, University of Minho, Campus Gualtar, 4710-057 Braga, Portugal. Tel.: (253) 604610. 


\section{Introduction}

Alcohol is the most widely used psychoactive substance in the world (World Health Organization, WHO, 2014). Binge drinking (BD), formally defined as the consumption of five or more drinks for men and four or more for women on the same occasion within a 2-h interval (National Institute of Alcohol Abuse and Alcoholism, NIAAA, 2004; Courtney and Polich, 2009), is a highly prevalent pattern of alcohol consumption in adolescents and young people in most Western countries. As such, around one out of three young Europeans and North Americans are binge drinkers (29\% of Europeans aged 15 to 25 years and 39\% of North Americans between 18 and 25 years) (Eurobarometer, 2010; SAMHSA, 2103), which has been associated with major social and health consequences such as unsafe sexual activity, motor vehicle crashes, violent behaviour, poor school performance, increased risk for cardiovascular impairments and hepatic injury, etc. (Brewer and Swahn, 2005; Goslawski et al., 2013; Llerena et al., 2015; Miller et al., 2007; Naimi et al., 2003; Valencia-Martín et al., 2008).

Adolescence and youth are periods of critical development in which the brain undergoes significant structural and functional changes (Casey et al., 2010; Giedd and Rapoport, 2010). These transformations in functioning and brain morphology mainly involve the prefrontal cortex (PFC) and other high-order association areas (Gogtay et al., 2004; Lebel and Beaulieu, 2011) and have been linked to meaningful enhancements in several cognitive processes such as attention, working memory, inhibitory control or decision making (Blakemore and Robbins, 2006; Hooper et al., 2004; Luna and Sweeney, 2004; Velanova et al., 2009; Yurgelun-Todd, 2007). Consequently, alcohol consumption during these periods of transition to adulthood is of particular concern given that excessive drinking might disrupt the neuromaturational processes of regions that are still maturing and therefore impair the cognitive functions partially supported by them (Squeglia et al., 2009).

Inhibitory control, i.e., the ability to suppress inappropriate responses or impulsive reactions with the aim of monitoring behaviour according to long-term goals (Allom et al., 2015; Diamond, 2013), deserves special attention given that this cognitive function has been related to individuals’ capacity to regulate alcohol consumption (Fillmore, 2003; Smith et al., 2014). Indeed, weak inhibitory control may predispose individuals to develop addictive behaviours, including alcohol abuse (López-Caneda et al., 2014a; Perry and Carrol, 2008). Specifically, poor response inhibition has been associated with BD in 
young social drinkers (Henges and Marczinski, 2012) as well as with more alcoholrelated problems and greater risk of alcohol dependence in adolescents (Nigg et al., 2006; Rubio et al., 2008). Likewise, acute alcohol intake may lead to impulse control deficits (Loeber and Duka, 2008; Rose and Duka, 2007) as well as to disruptions in brain functioning related to inhibitory control (Nikolau et al., 2013; Euser and Franken, 2012).

Young BDs have also shown lower performance on neuropsychological tests assessing inhibitory ability compared to age-matched controls (Czapla et al., 2014; Poulton et al., 2016). At the same time, electroencephalographic (EEG) recordings during Go/NoGo and Stop Signal tasks point to abnormalities in the event-related potentials (ERPs) linked to response inhibition in social and heavy drinkers (López-Caneda et al., 2012, 2014b; Petit et al., 2012; Smith and Mattick, 2013). These anomalies affect essentially the NoGo-P3 component, a positive waveform occurring between 300-700 ms after stimulus onset that has a maximum amplitude at fronto-central sites and has been functionally associated with response inhibition (Kok et al., 2004; Wessel and Aron, 2015). Thus, heavy social drinkers exhibited delayed NoGo-P3 latencies in alcoholrelated contexts, which was considered an index of prioritizing processing related to alcohol that might lead to inhibitory deficits (Petit et al., 2012). Furthermore, BDs also showed increased amplitude in NoGo-P3 and Stop-P3 (an analogous component to NoGo-P3 evoked during the Stop Signal task), suggesting the activation of additional/compensatory neural resources that would allow BDs to efficiently carry out the response inhibition (López-Caneda et al., 2012; Smith and Mattick, 2013).

There is considerable evidence suggesting that ERP waveforms emerge from superimposed neuroelectric oscillations induced by sensory or cognitive processes framed within dynamic ongoing EEG activity (Karakas et al., 2000; Klimesch et al., 2004). These oscillations, when analysed in the context of stimulus-related brain function, are frequently called event-related oscillations (EROs). The study of EROs enables the measurement (frequency-specific) of oscillatory activity in neural circuits that is temporally related to the sensory and cognitive processing of stimuli (Başar et al., 2001a). EROs are commonly classified according to the "natural frequencies" of the brain (Başar et al., 2001b), i.e., delta (0.5-4 Hz), theta (4-8 Hz), alpha (8-12 Hz), beta (12-30 Hz), and gamma (30-70 Hz). Despite their functional meanings often being task-specific, certain frequency bands within oscillatory responses may underlie different cognitive functions. In particular, research has shown a close relationship between delta and theta responses and inhibitory control processes evoked during the Go/NoGo paradigm (Harper et al., 
2014, 2016; Kirmizi-Alsan et al., 2006; Lavallee et al., 2014; Yamanaka and Yamamoto, 2010). However, the relationship between delta and theta oscillations and cognitive functions are not exclusive to response inhibition, as they have also been associated with a myriad of other cognitive processes such as perception, attention, error monitoring, memory and decision making (Cohen et al., 2009; Güntekin and Başar, 2016; Sauseng et al., 2010; Yordanova et al., 2004).

EROs have been shown to be sensitive to both normal and abnormal cognitive functioning in humans (Başar and Güntekin, 2013). Regarding alcoholism, several studies have reported attenuated delta and/or theta oscillations in alcohol-dependent patients during Go/NoGo tasks to both Go and NoGo stimuli, which has been interpreted as reflecting deficient inhibitory and attentional processing (Colrain et al., 2011; Kamarajan et al., 2004; Pandey et al., 2016). However, even though oscillatory responses have proven to be a useful tool for studying the neural response linked to inhibition in alcoholics, and that neural oscillations during resting states have been shown to be sensitive to the BD pattern (Correas et al., 2015, 2016; Courtney and Polich, 2010), to our knowledge there is no study that has evaluated EROs in young BDs. Bearing this in mind, the objective of this study was to determine whether young BDs would also exhibit impairments in oscillatory signals, particularly in delta and theta frequencies within the time window corresponding to Go- and NoGo-P3 components, i.e., the electrophysiological signals linked to execution and inhibition of a motor response. Furthermore, although our primary interest was in the EROs, we also examined the GoP3 and NoGo-P3 signals of the ERPs in order to compare time-domain and frequencydomain measures.

Given that this is the first study that directly compares EROs in these two groups, our a priori hypothesis is based on the results from alcohol-dependent subjects. Thus, we hypothesize that delta/theta oscillations linked to response inhibition will be modulated by the BD pattern. Specifically, we predict that young BDs will display reduced oscillatory activity in delta/theta frequency ranges during response inhibition processes as compared to age-matched controls with low or no alcohol consumption.

\section{Materials and Methods}

\subsection{Participants}


Seventy-two students from the Complutense University of Madrid (Spain) participated in the study. Participants were selected on the basis of their responses to a questionnaire that included the Spanish validated version of the Alcohol Use Disorder Identification Test (AUDIT) (Guillamón et al., 1999). Participants were asked to keep a record of daily alcohol consumption by indicating the type of drink, the quantity and the intensity of drinking. Blood alcohol concentration (BAC) was calculated based on the information of the drinking episodes of the last six months according to the following formula:

$$
B A C=\left(\frac{G}{W \times b w}\right)-m r \times D P
$$

where $G$ corresponds to grams of alcohol consumed on one occasion (the occasion of greatest consumption in the last month); $W$ is body weight (kilograms); $b w$ or body water is a constant related to the water content of the human body, with value 0.68 for males and 0.55 for females; $m r$ is the metabolization rate with a value of 0.15 for males and 0.18 for females; and $D P$ is the drinking period (hours). Taking into account the NIAAA's BD definition, where "a binge is a pattern of drinking alcohol that brings BAC to 0.08 grams per cent or above" (NIAAA, 2004), participants reaching BAC $\geq 0.08 \mathrm{~g} / \mathrm{dL}$ at least once during the last month were classified as BDs. On the other hand, the control group consisted of students who have never reached an alcohol concentration of 0.08 grams. Consequently, 36 participants were classified as BDs (19 females) and 36 as controls (16 females); 20 of the controls (8 females) were abstainers.

Impulsivity was assessed by the Barratt Impulsiveness Scale (BIS-11; Patton et al., 1995) and psychopathological symptoms were measured by the Symptom Checklist90 revised questionnaire (SCL-90-R; Derogatis, 1983). Likewise, participants were questioned about their use of other drugs such as tobacco, cannabis, cocaine, amphetamines and ecstasy (MDMA).

Exclusion criteria were: non-corrected sensory deficits, any episode of loss of consciousness for more than 20 minutes, history of traumatic brain injury or neurological disorder, personal history of psychopathological disorders (according to DSM-IV-TR criteria), family history of alcoholism or substance abuse in first degree relatives, consumption of medical drugs with psychoactive effects (e.g., sedatives or anxiolytics) during the week previous to the assessment, AUDIT scores $\geq 20$, and use of illegal drugs except cannabis.

\subsection{Procedure}


Participants were asked to abstain from consuming alcohol for at least $24 \mathrm{~h}$ before the experiment. They were submitted to a Breathalyzer test, and the assessment was only performed after verifying $0 \%$ breath alcohol level. Additionally, subjects were instructed not to smoke, or drink tea or coffee for at least $3 \mathrm{~h}$ before the assessment.

Participants performed a Go/NoGo task. They were instructed to fixate on a small cross located centrally on a LCD monitor. Squares or circles (green or blue) with a visual angle of $3^{\circ} \times 3^{\circ}$ were equiprobably presented (50/50) during $100 \mathrm{~ms}$ in the centre of the screen with an 1100-1500 ms interstimulus interval (offset-onset). Figures were presented in a randomized order in two series of 200-225 stimuli around four minutes each. Subjects had to press a button (50\% with their right hand and the other $50 \%$ with their left hand) as fast as possible to the Go trials (green circle and blue square) and not to respond to the NoGo trials (blue circle and green square).

All participants gave written informed consent and received monetary compensation for their participation. The experiment was undertaken in compliance with Spanish legislation and the Code of Ethical Principles for Medical Research Involving Humans Subjects outlined in the Declaration of Helsinki.

\subsection{Data Analysis}

\subsubsection{Analysis of behavioral and demographic data}

Only responses occurring between 100 and 1000 ms after the onset of a Go stimulus were considered to be correct responses. No-responses to NoGo stimuli were scored as correct inhibitions. Reaction time (RT) and percentage of correct responses and correct inhibitions were analysed by a Student's t-test for independent samples. The same statistical analysis was applied to demographic data.

\subsubsection{Electroencephalographic recording}

The electroencephalogram (EEG) was recorded using a 64-channel ActiCap system (Brain Products, Munich, Germany). Electrodes were Ag/AgCl active electrodes with integrated circuit of noise suppression and they were located according to the 10/10 system (American Clinical Neurophysiology Society, ACNS, 2006). All active electrodes were referred to the nose tip and grounded with an electrode placed at Fpz. Vertical and horizontal electrooculogram activity was recorded to control for potentials evoked by eye 
movements and blinks. According to impedance levels allowed by the ActiCap system, electrode impedances were kept below $20 \mathrm{k} \Omega$. EEG signals were continuously amplified and digitized at a rate of $500 \mathrm{~Hz}$, and filtered on-line with a $0.01-100 \mathrm{~Hz}$ band pass filter.

\subsubsection{Event-Related Potential Analysis}

For the ERP analysis, data were processed with BrainVision Analyzer software (Version 2.1). The EEG signal was corrected for vertical and horizontal ocular artifacts by the procedure developed by Gratton et al. (1983). It was then digitally filtered off-line with a 0.1-30 Hz bandpass filter and segmented into epochs of $1000 \mathrm{~ms}$ (from -100 to $900 \mathrm{~ms}$ ). Baseline correction was applied; epochs exceeding $\pm 80 \mu \mathrm{V}$ at any scalp electrode were rejected and EEG epochs corresponding to incorrect responses (omissions or false alarms) were excluded. The number of retained trials was similar across the two conditions (Go and NoGo) for both groups.

ERP waveforms were extracted by averaging across trials for each condition. The averaged ERPs were analysed with a semiautomatic peak detection procedure and subsequently reviewed and manually corrected for each of the midline electrode sites of interest (MESOIs) (Andrew and Fein, 2010), these being Fz, FCz, Cz, CPz, and Pz. The nature of the task (four different stimuli with equal-probability for each of them) entails a noteworthy difficulty for response selection, which may lead to two P3 subcomponents (Falkenstein et al., 1994, 1995; Fox et al., 2000). In the present study we measured both early-P3 and late-P3. These subcomponents were identified in the averaged waveforms elicited by Go and NoGo stimuli as the largest positive peak between 300- 450 ms (earlyP3) and between 500-600 ms (late-P3) after stimulus onset. Amplitude ( $\mu \mathrm{V})$ and latency (ms) values of both components were obtained for each of the MESOIs. A mixed-model ANOVA with two between-subject factors (Group: control and BD; Gender: male and female) and two within-subject factors (Condition: Go and NoGo; Electrode: five MESOIs) was used to examine the data separately for each subcomponent (alpha level $\leq$ 0.05). Where appropriate, degrees of freedom were corrected by the Greenhouse-Geisser estimate, and post hoc paired comparisons were performed with the Bonferroni adjustment for multiple comparisons, also with an alpha level $\leq 0.05$.

\subsubsection{Time-Frequency Analysis}

Along with ERP analysis, time-frequency analysis was carried out, which required some specific data pre-processing steps. For this analysis, the EEG signal was digitally filtered 
off-line with a $0.1-70 \mathrm{~Hz}$ bandpass filter and then corrected for ocular artifacts by the same procedure used for the ERPs. The EEG signal was re-referenced to the averaged reference and segmented into epochs of $2000 \mathrm{~ms}$, from $500 \mathrm{~ms}$ pre-stimulus to $1500 \mathrm{~ms}$ post-stimulus. Epochs exceeding $\pm 80 \mu \mathrm{V}$ at any scalp electrode were rejected and, as with the ERPs, EEG epochs corresponding to incorrect responses were excluded.

Time-frequency decomposition was performed on MATLAB R2015a (v8.5.0.197613, MathWorks) by first multiplying the result of the Fast Fourier Transform (FFT) of the EEG data by the FFT of different complex Morlet wavelets, and then computing the inverse FFT (iFFT) of the result. 32 Morlet wavelets were created in logarithmically spaced steps from $1 \mathrm{~Hz}$ to $40 \mathrm{~Hz}$, and with 3 cycles at the lowest frequency up to 10 at the highest frequency (also in logarithmically spaced steps). The iFFT yielded a complex estimate at each frequency $f$ and time point $t$ (with steps of $10 \mathrm{~ms}$ ). To obtain the power spectrogram, the squared absolute value of the complex estimate in each $t f$ point was computed. Resulting power values were normalized by transforming the power change of each $t f$ point to $\mathrm{dB}$, relative to the mean power in the baseline interval (from 300 to $-100 \mathrm{~ms}$ ) of each frequency, using the formula: $\mathrm{dB}_{\mathrm{tf}}=10 \log _{10}\left(\operatorname{signal}_{\mathrm{tf}} /\right.$ $\overline{\text { baselıne }_{\mathrm{f}}}$; where the bar over baseline indicates the mean of the baseline interval.

The resulting time-frequency values (total ERO power) were assessed statistically using the nonparametric cluster-based random permutation method (Maris \& Oostenveld 2007). The clustering used 4000 iterations and was performed on time-frequency data for each condition (Go and NoGo) and for each of the MESOIs included in the ERP analysis. To create a null-hypothesis distribution, subjects were randomized across groups in each iteration, and then two-tailed independent samples t-tests were performed for each timefrequency point. The cluster with the maximum absolute sum of t-values among all significant $(\mathrm{p}<.01)$ pixels was saved. When all permutations were completed, the $\mathrm{t}-$ values of the saved clusters were arranged in ascending order. A cluster was considered significant if the sum of t-values of significant pixels in the real t-tests was below the 2.5 percentile or above the 97.5 percentile in the sorted permutation t-values.

[INSERT TABLE 1 ABOUT HERE]

\section{Results}

\subsection{Demographic results}


Demographic data are summarized in Table 1. There were no significant differences between groups regarding age, handedness, regular use of cannabis (several times a week or every day), age of onset of regular use of alcohol, general severity index (GSI) of the SCL-R or BIS-11 scores (neither in total scores nor in the three second order factors: attentional, motor and nonplanning). Groups differed significantly in total AUDIT score $(p=.001)$, regular use of tobacco (several times a week or every day) $(p<.001)$, and $\operatorname{BAC}(p=.001)$.

\subsection{Behavioural results}

Behavioural data are summarized in Table 2. Groups did not differ in any of the behavioural variables analysed (RT, percentage of correct responses and percentage of correct inhibitions).

\section{[INSERT TABLE 2 ABOUT HERE]}

\subsection{ERP results}

The grand averages of ERPs for each group in both conditions are shown in Figure 1. Analysis of the early-P3 amplitude showed significant effects for Condition factor $[F(1,68)=42.26, p<.001]$, with larger amplitude in the Go than in the NoGo condition, and also for Electrode factor $[F(4,272)=56.53, p<.001]$, with the largest amplitude at Pz. Analysis of P3 latency only showed significant effects for Electrode factor $[F(4,272)$ $=8.15, p<.001]$, with the shortest latency at CPz. Regarding the late-P3, amplitude analysis only revealed significant effects for Condition $(\mathrm{F}(1,68)=4.3 ; \mathrm{p}=.042)$ and Electrode $(\mathrm{F}(4,272)=93.5 ; \mathrm{p}<.001)$ factors, showing larger amplitude in the Go than in the NoGo condition and the largest amplitude at Pz. Significant effects were also found in the latency analysis for the Condition $(F(1,68)=4.42 ; \mathrm{p}=.039)$ and Electrode $(F(4,272)$ $=4.23 ; \mathrm{p}=.002$ ) factors, showing longer latencies in the Go than in the NoGo condition and the shortest latency at Pz. There were no main effects or interactions involving group or sex in either amplitude or latency for any of the two subcomponents.

[INSERT FIGURE 1 ABOUT HERE]

\subsection{Time-frequency results}


The time-frequency representation and the energy curves for each group in both conditions are shown in Figures 2 and 3 respectively. Statistical analysis showed significant differences between groups during the Go condition in central and parietal locations. Specifically, BDs displayed lower total power in delta and theta frequencies compared to controls at $\mathrm{Cz}(p=.022$; time window $=260-660 \mathrm{~ms}$; frequency range $=2.8$ $8.0 \mathrm{~Hz}$ ) and $\mathrm{Pz}(p=.013$; time window $=440-900 \mathrm{~ms}$; frequency range $=2.3-5.5 \mathrm{~Hz}$ ) electrodes. Regarding the NoGo condition, significant effects were found in frontal and parietal locations. Specifically, BDs exhibited lower delta and theta power than controls at $\mathrm{Fz}(p=.004$; time window $=350-820 \mathrm{~ms}$; frequency range $=1.9-4.5 \mathrm{~Hz})$ and $\mathrm{Pz}(p=$ .002 ; time window $=350-900 \mathrm{~ms}$; frequency range $=2.6-7.3 \mathrm{~Hz}$ ) electrodes. In both conditions, the significant clusters showed maximal differences within the time range corresponding to Go-P3 and NoGo-P3 components, i.e., around 300-700 ms after the Go/NoGo signal onset (see Figure 2).

[INSERT FIGURE 2 ABOUT HERE]

[INSERT FIGURE 3 ABOUT HERE]

\section{Discussion}

This is the first study to examine oscillatory brain activity in young people with a BD pattern of alcohol consumption. Results reveal that BDs showed significantly lower delta and theta EROs during Go and NoGo conditions in comparison with the control group. These differences were localized in central and parietal regions for Go trials and in frontal and parietal regions for NoGo trials.

The results of the present study are consistent with previous ERO studies in chronic alcoholics during visual Go/NoGo tasks (Colrain et al., 2011; Kamarajan et al., 2004; Pandey et al., 2016), where significant differences between healthy controls and alcohol-dependent patients were observed in delta and theta oscillations during Go and NoGo conditions. In the first study, Kamarajan et al., (2004) reported reduced delta and theta oscillatory activity in abstinent chronic alcoholics that was more prominent in NoGo trials in the frontal region. The authors proposed that this decrease might be indicative of 
disruption of the neural circuit responsible for inhibitory control and that this deficit might constitute an endophenotypic marker for alcoholism. Another study from the same research group showed lower evoked delta, theta and alpha power in alcoholics compared to controls for Go and NoGo stimuli (Pandey et al., 2016). Again, the results were interpreted as suggestive of neurofunctional deficits during inhibition and execution of a response. Finally, Colrain et al., (2011) observed attenuated delta oscillations in alcoholics during response inhibition, which was related to reduced white matter integrity in the cingulate bundles. According to the authors, the lower delta power observed in alcoholics compared to controls might emerge as a consequence of degradation of frontoparietal pathways involved in inhibitory processing.

The present data examining EROs in young BDs extend previous findings reported in abstinent chronic alcoholics given that BDs, similarly to alcohol-dependent patients, displayed decreased delta and theta activity associated with response inhibition and response activation. Regarding these frequency bands, it has been argued that it is not possible to assign a single function to a given type of oscillatory activity since brain functions arise from series of superimposed oscillations in different frequency ranges (Başar et al., 2001a; Sauseng et al., 2007). Thus, delta and theta oscillations have been linked to multiple processes including perception, attention, signal detection, error monitoring, reward processing, memory, inhibition and decision making (Başar-Eroglu and Demiralp, 2001; Cohen et al., 2009; Güntekin and Başar, 2016; Marco-Pallarés et al., 2008; Sauseng et al., 2010; Yamanaka and Yamamoto, 2010; Yordanova et al., 2004). Even so, a number of studies have demonstrated that these frequency bands play an important and definite role in inhibitory control processes. Accordingly, increased delta and/or theta power has been reported for successful compared to failed inhibitions (Wessel and Aron, 2013, 2014) for withholding compared to execution of a response (Lavallee et al., 2014; Nigbur et al., 2011), as well as when a greater number of preceding Go stimuli are presented before the NoGo stimuli, which involve greater inhibitory effort (Harper et al., 2016). Likewise, delta and/or theta oscillations elicited by NoGo stimuli are reduced in subjects with high-risk for different disinhibitory spectrum disorders such as attention-deficit/hyperactivity disorder (Krämer et al., 2009) or alcoholism (Kamarajan et al., 2006), suggesting that weaker low-frequency oscillations linked to response inhibition may predispose individuals to develop alcoholism and/or other disinhibitory disorders. 
Based on these findings and the results reported from studies on alcoholdependent patients, it can be suggested that the reduced delta and theta power observed in the present study may reflect deficient oscillatory activity in young BDs during response inhibition. Furthermore, the finding that group differences were mostly detected within the time window corresponding to the NoGo-P3 - a component typically associated with motor inhibition (Smith et al., 2008; Wessel and Aron, 2015)- along with the fact that only the NoGo condition showed significantly lower delta and theta power in BDs compared to controls at the frontal region -the main region engaged in inhibitory control processes (Wiecki and Frank, 2013)- seems to strengthen the hypothesis that BD may be associated with functional anomalies in the neural oscillations linked to inhibitory responses.

Furthermore, this study has also shown abnormal oscillatory delta and theta activity in young BDs during response execution. Delta and theta power reductions in Go trials were localized in central and parietal sites and were maximal within the time range of Go-P3. These frequency bands have been considered the major contributors to the P3 signal (Başar-Eroglu et al., 1992; Demiralp et al., 2001). Go-P3 is functionally equivalent to the P3b component typically obtained in oddball tasks, which has been related to target detection involving both attention and memory processing (Polich, 2007). Thus, ERO anomalies in delta and theta oscillations during the Go condition might suggest dysfunctions in neurophysiological mechanisms underlying attentional and workingmemory processes, which is a common finding in chronic alcoholics (Jones et al., 2006; Pandey et al., 2016).

Likewise, several studies have demonstrated that BDs perform poorly in inhibition-related neuropsychological tests such as Go/NoGo (Czapla et al., 2014), StopSignal (Nederkoorn et al., 2009), Monetary Incentive Control (Poulton et al., 2016) and Stroop tasks (Hallgren et al., 2013), indicating weaker inhibitory ability in subjects with a BD pattern compared to age-matched controls. In turn, some ERP studies evaluating inhibitory control in young BDs seem to point to functional disruptions in the neural responses associated with response inhibition. As such, anomalies in latency (Petit et al. 2012) and amplitude (López-Caneda et al., 2012; Smith and Mattick, 2013) of NoGo-P3 and Stop-P3 have been observed in young binge and social drinkers while trying to withhold a response.

Nevertheless, not all studies conducted on BDs have found these effects, since some recent reports (using also a visual GoNoGo task) have failed to show group 
differences in these electrophysiological components linked to inhibition and execution responses (Watson et al., 2014, 2016). The present study also failed to find differences in Go- and NoGo-P3 responses, i.e., these components seemed not to be significantly affected by BD, which is contrary to what was observed in a previous study from our laboratory with a different sample (López-Caneda et al., 2012). One possible explanation for the absence of significant differences between groups in the Go- and NoGo-P3 components concerns the fact that subjects in our first study had been drinking alcohol for a longer time than BDs of the present study. In this regard, anomalies in NoGo-P3 only emerged after more than two years maintaining the BD pattern (López-Caneda et al., 2012). Thus, it is possible that the present study has failed to show time-domain differences between groups because impairments in ERPs might not be apparent in early stages of BD. However, electrophysiological differences might emerge when frequencydomain measures are used. In this vein, studies on offspring of alcoholics and highalcohol preferring mice have shown stronger differences between groups for ERO signals than for P3 amplitude (Criado and Ehlers, 2009; Rangaswamy et al., 2007), suggesting that ERO measures are a more stable and useful marker in the study of alcoholism and related disorders (Gilmore et al., 2010; Pandey et al., 2012). Altogether, it can be suggested that ERO analysis may be able to detect electrophysiological anomalies linked to $\mathrm{BD}$ in youths when performing a Go/NoGo task that are not identifiable by standard time-domain analyses.

An additional question that arises from the present results is why ERO measures might provide unique information beyond the ERP amplitude measures. While ERPs contain information about time- and phase-locked activity, total ERO power measures contain contributions from both phase-locked and nonphase-locked activity and, consequently, provide independent information to that obtained from the ERP amplitude measures (Pfurtscheller and Lopes da Silva, 1999). Thus, these nonphase-locked oscillations -also known as induced activity or event-related synchronization/desynchronization (ERS/ERD)-, which cannot be extracted by traditional time-domain analyses, might account for the group differences observed in the present study. This possibility has been already reported from studies with alcoholdependent patients and with offspring of alcoholics, from which has been proposed that ERO measures may provide additional or even more sensitive group discriminators than the ERPs (Andrew and Fein, 2010; Jones et al., 2006; Rangaswamy et al., 2007). 
However, further inquiries regarding EROs in BD population are required to address this issue.

Finally, this study has some limitations that encourage us to be cautious when interpreting the results. On one hand, the limited sample size (although suitable for an EEG study) could undermine the reliability of results. Therefore additional research must be conducted in order to verify or refute the present results. On the other hand, the nature of this study does not allow us to determine whether these anomalies in EROs precede the BD pattern or, conversely, arise as a consequence of heavy alcohol drinking. In this sense, studies on offspring of alcoholics have found that such high-risk youths exhibit decreased power in delta and/or theta bands during the GoNoGo task (Kamarajan et al., 2006), as well as during oddball (Rangaswamy et al., 2007) and gambling (Kamarajan et al., 2015) tasks, suggesting that these effects might constitute a biological marker of vulnerability to alcoholism, rather than being a consequence of alcohol consumption. However, in the present study, in which individuals with a family history of alcoholism were excluded and where no differences were found among groups in impulsivity (as measured by BIS-11), no anomalous ERPs or EROs before the onset of alcohol consumption were expected, although longitudinal studies including future BDs without prior alcohol consumption are needed to test this hypothesis.

In summary, the present study is the first ERO study to report abnormal neural activity related to response execution and inhibition during a Go/NoGo task in young BDs. Specifically, this study showed weaker oscillatory responses in delta and theta bands during both response inhibition and response execution in the BD group as compared to age-matched control group mainly within the time window of 300-700 ms poststimulus. This finding is congruent with previous ERO studies in chronic alcoholics using visual Go/NoGo tasks, where lower delta and/or theta oscillations were reported in alcoholdependent patients compared to healthy controls (Colrain et al., 2011; Kamarajan et al., 2004; Pandey et al., 2016). Thus, BDs appear to show disruptions in neural oscillations similar to those observed in subjects with alcohol dependence. The reduced delta and theta EROs might reflect impairments in the neural circuit involved in both the activation and inhibition of a response. This outcome might constitute a new manisfestation of functional anomalies in inhibitory control and attentional/working memory processes in BDs, which could not be disclosed by means of traditional time-domain methods. These findings are particularly valuable since they are the first to evidence that oscillatory brain 
activity may be a sensitive indicator of underlying brain anomalies in young BDs which could complement standard ERP measures.

\section{Acknowledgements}

This study was supported by the projects SPI/2010/134 and SPI/2010/051 from the Spanish Ministry of Health and Social Politics (National Plan on Drugs). Eduardo LópezCaneda was supported by the SFRH/BPD/109750/2015 Postdoctoral Fellowship of the Portuguese Foundation for Science and Technology as well as by the Psychology Research Centre (UID/PSI/01662/2013), co-financed by FEDER through COMPETE2020 under the PT2020 Partnership Agreement (POCI-01-0145-FEDER007653). Carina Carbia was supported through the FPU program (FPU2013-04569) of the Spanish Ministry of Education, Culture and Sports.

\section{References}

Allom V, Mullan B, Hagger M (2015) Does inhibitory control training improve health behaviour? A meta-analysis. Health Psychol Rev 10:1-19.

American Clinical Neurophysiology Society (2006) Guideline 5: guidelines for standard electrode position nomenclature. Am J Electroneurodiagnostic Technol 46:222225.

Andrew C and Fein G (2010) Event-Related Oscillations Versus Event-Related Potentials in a P300 Task as Biomarkers for Alcoholism. Alcohol Clin Exp Res 34:669-680.

Başar E, Başar-Eroglu C, Karakaş S, Schürmann M (2001a) Gamma, alpha, delta, and theta oscillations govern cognitive processes. Int J Psychophysiol 39:241-8.

Basar E and Guntekin B (2013) Review of delta, theta, alpha, beta, and gamma response oscillations in neuropsychiatric disorders. Suppl Clin Neurophysiol 2013 62:303341. 
Başar E., Schürmann M., Demiralp T., Başar-Eroglu C., Ademoglu A (2001b) Eventrelated oscillations are 'real brain responses'-wavelet analysis and new strategies. Int J Psychophysiol 39: 91-127.

Başar-Eroglu C, Başar E, Demiralp T, Schürmann M (1992) P300-response: possible psychophysiological correlates in delta and theta frequency channels. A review. Int J Psychophysiol 13:161-179.

Başar-Eroglu C and Demiralp T. (2001) Event-related theta oscillations: an integrative and comparative approach in the human and animal brain. Int J Psychophysiol 39:167-195.

Blakemore SJ and Robbins TW (2012) Decision-making in the adolescent brain. Nat Neurosci 15:1184-1191.

Brewer RD and Swahn MH (2005) Binge drinking and violence. JAMA 294:616-618.

Casey BJ, Duhoux S, Cohen MM (2010) Adolescence: what do transmission, transition, and translation have to do with it? Neuron 67:749-760.

Cohen MX, Elger CE, Fell J (2009) Oscillatory activity and phase-amplitude coupling in the human medial frontal cortex during decision making. J Cogn Neurosci 21:390402.

Colrain IM, Sullivan EV, Ford JM, Mathalon DH, McPherson SL, Roach BJ et al. (2011) Frontally mediated inhibitory processing and white matter microstructure: age and alcoholism effects. Psychopharmacology 213:669-679.

Correas A, Cuesta P, López-Caneda E, Rodríguez Holguín S, García-Moreno LM, Pineda-Pardo JA, et al. (2016) Functional and structural brain connectivity of young binge drinkers: a follow-up study. Sci Rep 6:31293.

Correas A, Rodríguez Holguín S, Cuesta P, López-Caneda E, García-Moreno LM, Cadaveira F, et al. (2015) Exploratory analysis of power spectrum and functional connectivity during resting state in young binge drinkers: A MEG study. Int J Neural Syst 25:1550008. 
Courtney KE and Polich J (2009) Binge drinking in young adults: Data, definitions, and determinants. Psychol Bull 135:142-156.

Courtney KE and Polich J (2010) Binge drinking effects on EEG in young adult humans. Int J Environ Res Public Health 7:2325-2336.

Criado JR and Ehlers CL (2009) Event-related oscillations as risk markers in genetic mouse models of high alcohol preference. Neuroscience 163:506-523.

Czapla M, Simon JJ, Friederich HC, Herpertz SC, Zimmermann P, Loeber S (2014) Is binge drinking in young adults associated with an alcohol-specific impairment of response inhibition? Eur Addict Res 2:105-113.

Demiralp T, Ademoglu A, Comerchero M, Polich, J (2001) Wavelet analysis of P3a and P3b. Brain Topogr 13:251-267.

Derogatis LR (1983) Administration, Scoring and Procedures Manual II for the Revised Version of the SCL-90. Baltimore: Johns Hopkins University Press.

Diamond A (2013) Executive functions. Annu Rev Psychol 64:135-168

Eurobarometer (2010) EU citizens’ attitudes towards alcohol. Special Eurobarometer 331 / Wave 72.3. Brussels: TNS Opinion y Social at the request of Directorate General Health and Consumers and coordinated by the Directorate-General for Communication of European Commission. http://ec.europa.eu/health/alcohol/docs/ebs_331_en.pdf

Euser AS and Franken IH (2012) Alcohol affects the emotional modulation of cognitive control: an event-related brain potential study. Psychopharmacology 222:459476.

Falkenstein M, Hohnsbein J, Hoormann J (1994) Effects of choice complexity on different subcomponents of the late positive complex of the event-related potential. Electroencephalogr Clin Neurophysiol 92:148-160. 
Falkenstein M, Koshlykova NA, Kiroj VN, Hoormann J, Hohnsbein J (1995) Late ERP components in visual and auditory Go/Nogo tasks. Electroencephalogr Clin Neurophysiol 96:36-43.

Fillmore MT (2003) Drug abuse as a problem of impaired control: current approaches and findings. Behav Cogn Neurosci Rev 2:179-197.

Fox AM, Michie PT, Wynne CDL, Maybery MT (2000) ERP correlates of response inhibition to elemental and configural stimuli in a negative patterning task. Clin Neurophysiol 111:1045-1053.

Giedd JN and Rapoport JL (2010) Structural MRI of pediatric brain development: what have we learned and where are we going? Neuron 67:728-734.

Gilmore CS, Malone SM, Bernat EM, Iacono WG (2010) Relationship between the P3 event-related potential, its associated time-frequency components, and externalizing psychopathology. Psychophysiology 47:123-132.

Gogtay N, Giedd JN, Lusk L, Hayashi KM, Greenstein D, Vaituzis A et al. (2004) Dynamic mapping of human cortical development during childhood through early adulthood. Proc Natl Acad Sci USA 101:8174-8179.

Goslawski M, Piano MR, Bian JT, Church E, Szczurek M, Phillips SA (2013) Binge drinking impairs vascular function in young adults. J Am Coll Cardiol 62:201207.

Gratton G, Coles MG, Donchin E (1983)A new method for off-line removal of ocular artifact. Electroencephalogr Clin Neurophysiol 55:468-484.

Guillamón MC, Solé AG, Farran JC (1999) Test para la identificación de transtornos por uso de alcohol (audit): traducción y validación del audit al catalán y castellano. Adicciones 11:337-347.

Güntekin B and Başar E (2016) Review of evoked and event-related delta responses in the human brain. Int J Psychophysiol 103:43-52. 
Hallgren KA and McCrady BS (2013) Interference in the alcohol Stroop task with college student binge drinkers. J Behav Health 2:112-119.

Harper J, Malone SM, Bachman MD, Bernat EM (2016) Stimulus sequence context differentially modulates inhibition-related theta and delta band activity in a go/nogo task. Psychophysiology 53:712-22.

Harper J, Malone SM, Bernat EM (2014) Theta and delta band activity explain N2 and P3 ERP component activity in a go/no-go task. Clin Neurophysiol 125:124-132.

Henges AL and Marczinski CA (2012) Impulsivity and alcohol consumption in young social drinkers. Addict Behav 37:217-220.

Hooper CJ, Luciana M, Conklin HM, Yarger RS (2004) Adolescents' performance on the Iowa Gambling Task: implications for the development of decision making and ventromedial prefrontal cortex. Dev Psychol 40:1148-1158.

Jones KA, Porjesz B, Chorlian D, Rangaswamy M, Kamarajan C, Padmanabhapillai A, et al (2006) S-transform time-frequency analysis of P300 reveals deficits in individuals diagnosed with alcoholism. Clin Neurophysiol, 117:2128-2143.

Kamarajan C, Pandey AK, Chorlian DB., Manz N, Stimus AT, Anokhin AP (2015) Deficient Event-Related Theta Oscillations in Individuals at Risk for Alcoholism: A Study of Reward Processing and Impulsivity Features. PLoS One 10:e0142659.

Kamarajan C, Porjesz B, Jones KA, Choi K, Chorlian DB, Padmanabhapillai A, et al (2004) The role of brain oscillations as functional correlates of cognitive systems: a study of frontal inhibitory control in alcoholism. Int J Psychophysiol 51:155180.

Kamarajan C, Porjesz B, Jones K, Chorlian D, Padmanabhapillai A, Rangaswamy M et al (2006) Event-related oscillations in offspring of alcoholics: neurocognitive disinhibition as a risk for alcoholism. Biol Psychiatry 59:625-634.

Karakaş S, Erzengin ÖU, Başar E (2000) The genesis of human event-related responses explained through the theory of oscillatory neural assemblies. Neurosci Lett 285:45-48. 
Kirmizi-Alsan E, Bayraktaroglu Z, Gurvit H, Keskin YH, Emre M, Demiralp T (2006) Comparative analysis of event-related potentials during Go/NoGo and CPT: decomposition of electrophysiological markers of response inhibition and sustained attention. Brain Res 1104:114-128.

Klimesch W, Schack B, Schabus M, Doppelmayr M, Gruber W, Sauseng, P (2004) Phaselocked alpha and theta oscillations generate the P1-N1 complex and are related to memory performance. Brain Res Cogn Brain Res 19:302-316.

Kok A, Ramautar JR, De Ruiter MB, Band GP, Ridderinkhof KR (2004) ERP components associated with successful and unsuccessful stopping in a stop-signal task. Psychophysiology 41:9-20.

Krämer UM, Rojo N, Schüle R, Cunillera T, Schöls L, Marco-Pallarés J, et al (2009) ADHD candidate gene (DRD4 exon III) affects inhibitory control in a healthy sample. BMC Neurosci 10:1.

Lavallee CF, Meemken MT, Herrmann CS, Huster RJ (2014) When holding your horses meets the deer in the headlights: time-frequency characteristics of global and selective stopping under conditions of proactive and reactive control. Front Hum Neurosci 8:994.

Lebel C and Beaulieu C (2011) Longitudinal development of human brain wiring continues from childhood into adulthood. J Neurosci 31:10937-10947.

Llerena S, Arias-Loste MT, Puente A, Cabezas J, Crespo J, Fábrega E (2015) Binge drinking: Burden of liver disease and beyond. World J Hepatol 7:2703-2715.

Loeber S and Duka T. (2008) Acute alcohol impairs conditioning of a behavioural reward-seeking response and inhibitory control processes-implications for addictive disorders. Addiction 104:2013-2022.

López-Caneda E, Cadaveira F, Crego A, Gómez-Suárez A, Corral M, Parada M, et al. (2012) Hyperactivation of right inferior frontal cortex in young binge drinkers during response inhibition: a follow-up study. Addiction 107:1796-1808. 
López-Caneda E, Rodríguez Holguín S, Cadaveira F, Corral M, Doallo S (2014a) Impact of alcohol use on inhibitory control (and vice versa) during adolescence and young adulthood: a review. Alcohol Alcohol 49:173-181.

López-Caneda E, Rodríguez Holguín S, Corral M, Doallo S, Cadaveira F (2014b) Evolution of the binge drinking pattern in college students: Neurophysiological correlates. Alcohol 48:407-418.

Luna B and Sweeney JA (2004) The emergence of collaborative brain function: FMRI studies of the development of response inhibition. Ann N Y Acad Sci 1021:296309.

Marco-Pallarés J, Cucurell D, Cunillera T, García R, Andrés-Pueyo A, Münte TF, Rodríguez-Fornells A (2008) Human oscillatory activity associated to reward processing in a gambling task. Neuropsychologia 46:241-248.

Maris E and Oostenveld R (2007) Nonparametric statistical testing of EEG-and MEGdata. J Neurosci Methods 164:177-190.

Maurage P, Joassin F, Speth A, Modave J, Philippot P, Campanella, S (2012) Cerebral effects of binge drinking: respective influences of global alcohol intake and consumption pattern. Clin Neurophysiol 123:892-901.

Miller JW, Naimi TS, Brewer RD, Jones SE (2007) Binge drinking and associated health risk behaviors among high school students. Pediatrics 119:76-85.

Naimi TS, Lipscomb LE, Brewer RD, Gilbert BC (2003) Binge drinking in the preconception period and the risk of unintended pregnancy: implications for women and their children. Pediatrics 111(Supp 1):1136-1141.

National Institute of Alcohol Abuse and Alcoholism (2004) NIAAA council approves definition of binge drinking. IAAA Newsletter, 3, p. 3. http://pubs.niaaa.nih.gov/publications/Newsletter/winter2004/Newsletter_Numb er3.pdf 
Nederkoorn C, Baltus M, Guerrieri R, Wiers RW (2009) Heavy drinking is associated with deficient response inhibition in women but not in men. Pharmacol Biochem Behav 93:331-336.

Nigbur R, Ivanova G, Stürmer B (2011) Theta power as a marker for cognitive interference. Clin Neurophysiol 122:2185-2194.

Nigg JT, Wong MM, Martel MM, Jester JM, Puttler LI, Glass JM, et al (2006) Poor response inhibition as a predictor of problem drinking and illicit drug use in adolescents at risk for alcoholism and other substance use disorders. J Am Acad Child Adolesc Psychiatry 45:468-475.

Nikolaou K, Critchley H, Duka T (2013) Alcohol affects neuronal substrates of response inhibition but not of perceptual processing of stimuli signalling a stop response. PloS One 8:e76649.

Pandey AK, Kamarajan C, Rangaswamy M, Porjesz B (2012) Event-Related Oscillations in Alcoholism Research: A Review. J Addict Res Ther S7:001

Pandey AK, Kamarajan C, Manz N, Chorlian DB, Stimus A, Porjesz B (2016) Delta, theta, and alpha event-related oscillations in alcoholics during Go/NoGo task: Neurocognitive deficits in execution, inhibition, and attention processing. Prog Neuropsychopharmacol Biol Psychiatry 65:158-171.

Patton JH, Stanford MS, Barratt ES (1995) Factor structure of the Barratt Impulsiveness Scale. J Clin Psychol 51:768-774.

Perry JL and Carroll ME (2008) The role of impulsive behavior in drug abuse. Psychopharmacology 200:1-26.

Petit G, Kornreich C, Noël X, Verbanck P, Campanella S (2012) Alcohol-related context modulates performance of social drinkers in a visual Go/No-Go task: a preliminary assessment of event-related potentials. PLoS One 7:e37466.

Polich J (2007) Updating P300: an integrative theory of P3a and P3b. Clin Neurophysiol, 118:2128-2148. 
Poulton A, Mackenzie C, Harrington K, Borg S, Hester R (2016) Cognitive control over immediate reward in binge alcohol drinkers. Alcohol Clin Exp Res 40:429-437.

Pfurtscheller G and Da Silva FL (1999) Event-related EEG/MEG synchronization and desynchronization: basic principles. Clin Neurophysiol 110:1842-1857.

Rangaswamy M, Jones KA, Porjesz B, Chorlian DB, Padmanabhapillai A, Kamarajan C, et al (2007) Delta and theta oscillations as risk markers in adolescent offspring of alcoholics. Int J Psychophysiol 63:3-15.

Rose AK and Duka T (2007) The influence of alcohol on basic motoric and cognitive disinhibition. Alcohol Alcohol 42:544-551.

Rubio G, Jiménez M, Rodríguez-Jiménez R, Martínez I, Ávila C, Ferre F et al (2008) The Role of Behavioral Impulsivity in the Development of Alcohol Dependence: A 4Year Follow-Up Study. Alcohol Clin Exp Res 32:1681-1687.

Sauseng P, Griesmayr B, Freunberger R, Klimesch W (2010) Control mechanisms in working memory: a possible function of EEG theta oscillations. Neurosci Biobehav Rev 34:1015-1022.

Sauseng P, Klimesch W, Gruber WR, Hanslmayr S, Freunberger R, Doppelmayr M (2007) Are event-related potential components generated by phase resetting of brain oscillations? A critical discussion. Neuroscience 146:1435-1444.

Smith JL, Johnstone SJ, Barry RJ (2008) Movement-related potentials in the Go/NoGo task: the P3 reflects both cognitive and motor inhibition. Clin Neurophysiol 119:704-714.

Smith JL and Mattick RP (2013) Evidence of deficits in behavioural inhibition and performance monitoring in young female heavy drinkers. Drug Alcohol Depend 133:398-404.

Smith JL, Mattick RP, Jamadar SD, Iredale JM (2014) Deficits in behavioural inhibition in substance abuse and addiction: a meta-analysis. Drug Alcohol Depend 145:133. 
Squeglia LM, Jacobus J, Tapert SF (2009) The influence of substance use on adolescent brain development. Clin EEG Neurosci 40:31-38.

Substance Abuse and Mental Health Services Administration (2013) Results from the 2012 ational Survey on Drug Use and Health: Summary of ational Findings, NSDUH Series H-46, HHS Publication No. (SMA) 13-4795. Rockville, MD: Substance Abuse and Mental Health Services Administration. http://www.samhsa.gov/data/NSDUH/2012SummNatFindDetTables/NationalFi ndings/ NSDUHresults2012.htm

Townshend JM and Duka T (2005) Binge drinking, cognitive performance and mood in a population of young social drinkers. Alcohol Clin Exp Res 29:317-325.

Valencia-Martín JL, Galán I, Rodríguez-Artalejo F (2008) The joint association of average volume of alcohol and binge drinking with hazardous driving behaviour and traffic crashes. Addiction 103:749-757.

Velanova K, Wheeler ME, Luna B (2009) The maturation of task set-related activation supports late developmental improvements in inhibitory control. J Neurosci 29:12558-12567.

Watson TD, Newton-Mora M, Pirkle J (2016) Event-related potential correlates of processing alcohol-related pictures in young adult binge drinkers. Am J Drug Alcohol Abuse 42:77-87.

Watson TD, Sweeney JF, Louis H (2014) Neurocognitive, psychological and behavioral correlates of binge drinking and use of alcohol with caffeinated beverages in college-aged adults. Am J Drug Alcohol Abuse 40:58-66.

Wessel JR and Aron AR (2014) Inhibitory motor control based on complex stopping goals relies on the same brain network as simple stopping. NeuroImage 103:225-234.

Wessel JR and Aron AR (2015) It's not too late: The onset of the frontocentral P3 indexes successful response inhibition in the stop-signal paradigm. Psychophysiology $52: 472-480$. 
Wessel JR and Aron AR (2013) Unexpected events induce motor slowing via a brain mechanism for action-stopping with global suppressive effects. J Neurosci 33:18481-18491.

Wiecki TV and Frank MJ (2013). A computational model of inhibitory control in frontal cortex and basal ganglia. Psychol Rev 120:329.

World Health Organization (2014) Global Status Report on Alcohol and Health. Geneva: World Health Organisation.

Yamanaka K and Yamamoto Y (2010) Single-trial EEG power and phase dynamics associated with voluntary response inhibition. J Cogn Neurosci 22:714-727.

Yordanova J, Falkenstein M, Hohnsbein J, Kolev V (2004) Parallel systems of error processing in the brain. Neuroimage 22:590-602.

Yurgelun-Todd D (2007) Emotional and cognitive changes during adolescence. Curr Opin Neurobiol 17:251-257. 


\section{Tables}

Table 1. Demographic and drinking characteristics of the Control and Binge Drinking groups.

\begin{tabular}{|c|c|c|}
\hline & Controls & Binge Drinkers \\
\hline $\mathrm{N}$ (females) & $36(19)$ & $36(16)$ \\
\hline Age & $18.08 \pm 0.28$ & $18.08 \pm 0.28$ \\
\hline $\begin{array}{c}\text { Handedness } \\
\text { (right/ambidextrous/left) }\end{array}$ & $32 / 3 / 1$ & $33 / 2 / 1$ \\
\hline Caucasian ethnicity (\%) & 100 & 100 \\
\hline Regular tobacco smokers & 0 & $6 *$ \\
\hline Regular use of cannabis & 0 & 0 \\
\hline $\begin{array}{c}\text { Age of onset of regular } \\
\text { drinking }\end{array}$ & $16.56 \pm 1.12$ & $14.72 \pm 1.18$ \\
\hline $\begin{array}{l}\text { Blood alcohol concentration } \\
\text { (BAC) in a drinking day } \\
\text { (g/dL) }\end{array}$ & $0.01 \pm 0.02$ & $0.17 \pm 0.09 *$ \\
\hline BIS-11 total score & $60.19 \pm 8.73$ & $63.25 \pm 9.12$ \\
\hline GSI score & $0.30 \pm 0.17$ & $0.30 \pm 0.14$ \\
\hline Total AUDIT score & $0.94 \pm 1.44$ & $7.44 \pm 1.29 *$ \\
\hline
\end{tabular}

$* \mathrm{p} \leq .05$ significant differences between groups. 
Table 2. Performance scores in the Control and Binge Drinking groups (mean \pm SD).

\begin{tabular}{ccc}
\hline Behavioral Performance & Controls & Binge Drinkers \\
\hline Response Time (ms) & $489.8 \pm 49.19$ & $512 \pm 60.06$ \\
\% Correct Responses & $95.93 \pm 5.42$ & $95.14 \pm 3.96$ \\
\% Correct Inhibitions & $90.79 \pm 5.42$ & $92.28 \pm 5.65$ \\
\hline
\end{tabular}




\section{Figure Legends}

Fig. 1 Grand average of event-related potentials for Control (blue line) and Binge Drinking (red line) groups in response to Go and NoGo stimuli. Averages are presented for $\mathrm{Fz}, \mathrm{Cz}$ and $\mathrm{Pz}$ electrodes.

Fig. 2 Time-frequency representation of Go and NoGo conditions for Control and Binge Drinking groups at $\mathrm{Cz}$ and $\mathrm{Pz}$ (Go condition) and $\mathrm{Fz}$ and $\mathrm{Pz}$ (NoGo condition) electrodes. The area embedded within each frame represents the frequency ranges and the time window that showed significant between-group differences.

Fig. 3 Energy curves of delta and theta power during Go and NoGo conditions. Curves correspond to the grand averages of the mean power values in delta and theta frequency bands. Data are presented for electrodes with significant between-group differences: $\mathrm{Cz}$ and Pz (Go condition); Fz and Pz (NoGo condition). 